18 Oct 2018 6:51 p.m.

FTUAM 04-02

hep-ph/0402285

\title{
The hadronic contributions to the anomalous magnetic moment of the muon
}

\author{
J. F. de Trocóniz and F. J. Ynduráin \\ Departamento de Física Teórica, C-XI \\ Universidad Autónoma de Madrid, \\ Canto Blanco, \\ E-28049, Madrid, Spain.
}

\begin{abstract}
We present a new, completely revised calculation of the muon anomalous magnetic moment, $a_{\mu}=\left(g_{\mu}-2\right) / 2$, comparing it with the more recent experimental determination of this quantity; this furnishes an important test of theories of strong, weak and electromagnetic interactions. These theoretical and experimental determinations give the very precise numbers,

$10^{11} \times a_{\mu}= \begin{cases}116591806 \pm 50 \pm 10(\mathrm{rad} .) \pm 30(\ell \times \ell) & \text { [Th., no } \tau] \\ 116591889 \pm 49 \pm 10(\mathrm{rad} .) \pm 30(\ell \times \ell) & \text { [Theory, } \tau] \\ 116592080 \pm 60 \quad \text { [Experiment]. } & \end{cases}$

In the theoretical evaluations, the first quantity does not, and the second one does, use information from $\tau$ decay. The first errors for the theoretical evaluations include statistical plus systematic errors; the other ones are the estimated errors due to incomplete treatment of radiative corrections and the estimated error in the light-by-light scattering contribution. We thus have a significant mismatch between theory and experiment. We also use part of the theoretical calculations to give a precise evaluation of the electromagnetic coupling on the $Z$, $\bar{\alpha}_{\text {Q.E.D. }}\left(M_{Z}^{2}\right)$, of the masses and widths of the (charged and neutral) rho resonances, of the scattering length and effective range for the $\mathrm{P}$ wave in $\pi \pi$ scattering, and of the quadratic radius and second coefficient of the pion form factor.
\end{abstract}


Typeset with $\mathrm{P}_{\mathrm{H}} \mathrm{ys}^{\mathrm{Ma}} \mathrm{T}_{\mathrm{E}} \mathrm{X}$ 
THE HADRONIC CONTRIBUTIONS TO THE ANOMALOUS MAGNETIC MOMENT OF THE MUON

\section{Introduction}

The anomalous magnetic moments of electrons and muons provide one of the more impressive tests of the standard model of strong, weak and electromagnetic interactions. The electron anomaly, $a_{e}$, receives only marginal contributions from weak and strong interactions, being dominated by electromagnetic (QED) radiative corrections. The agreement between theory and experiment is such that, if we turn it around, the experimental value of $a_{e}$ provides the more precise determination of the fine structure constant, ${ }^{[1]} \alpha$.

For the muon magnetic moment, the recent precise measurements ${ }^{[2]}$ of the muon anomaly, $a_{\mu}$, constitute one of the more impressive tests not only of electroweak interactions, but of strong interactions as well. After these measurements, the world average value for $a_{\mu}=\left(g_{\mu}-2\right) / 2$ is

$$
10^{11} \times a_{\mu}=116592080 \pm 60 \quad \text { [Experiment]. }
$$

Considering that theory also gives the value of the magnetic moment itself (and not only the anomaly) the agreement of theory and experiment that we will describe represents a precision of one or two parts in a billion. Nevertheless, and as we will see, there remains a discrepancy at the level of $2.3 \sigma$ to $3.3 \sigma$.

The electromagnetic and weak contributions to $a_{\mu}$ have been calculated with great accuracy: ${ }^{[3]}$

$$
\begin{array}{rrr}
10^{11} \times a_{\mu}(\mathrm{QED}) & =116584719 \pm 1.8, \\
10^{11} \times a_{\mu}(\text { Weak }) & = & 152 \pm 3 .
\end{array}
$$

Combining this with (1.1), we find the experimental number for the hadronic contributions to $a_{\mu}$,

$$
\left.\left.10^{11} \times a_{\mu} \text { (Hadr. }\right)=7209 \pm 60 \quad \text { [Experiment }\right] .
$$

The evaluation of this quantity, $a_{\mu}$ (Hadr.), from theory will be the main subject of the present note; we will find

$$
\left.10^{11} \times a_{\mu} \text { (Hadr. }\right)= \begin{cases}6935 \pm 50 \pm 10(\text { rad. }) \pm 30(\ell \times \ell) & {[\text { No } \tau]} \\ 7018 \pm 49 \pm 10(\text { rad. }) \pm 30(\ell \times \ell) & {[\text { With } \tau]}\end{cases}
$$

The numbers above depend on whether or not one includes information on $\tau$ decay, which is probably the more reliable result as it is the one that incorporates more information: see our text below for details. In the errors, "rad" and " $\ell \times \ell$ " refer, respectively, to estimated errors due to uncalculated radiative corrections and light-by-light scattering contributions. The results in (1.4) takes into account all the more recent $e^{+} e^{-}$annihilations data, and $e \pi$ scattering data. At the end of the present article we will comment on the degree of agreement of theory and experiment, compare our results with those of other recent calculations, and discuss the possible reasons for the discrepancy between (1.3) and (1.4).

We will also give a summary of the results that some of the calculations imply for the electromagnetic coupling on the $Z, \bar{\alpha}_{\text {Q.E.D. }}\left(M_{Z}^{2}\right)$, for the masses and widths of the (charged and neutral) rho resonances, for the scattering length and effective radius for the $\mathrm{P}$ wave in pion-pion scattering, and for the quadratic radius and second coefficient of the pion form factor. 


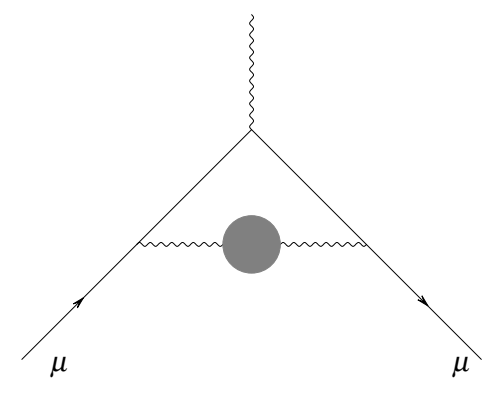

Figure 1. The order $\alpha^{2}$ hadronic contributions to the muon magnetic moment. The blob represents an arbitrary hadronic state. The wavy lines are photons.

\section{The hadronic contributions to $a_{\mu}$. I: the $O\left(\alpha^{2}\right)$ piece}

To order $\alpha^{2}$, the contributions to $a_{\mu}$ (Hadr.) can be represented by the diagram shown in Fig. 1. As has been known for a long time, they can be written in terms of the cross section for $e^{+} e^{-}$annihilation into hadrons, as follows. We first write

$$
\begin{aligned}
a_{\mu}^{(2)}(\text { Hadr. }) & =12 \pi \int_{4 m_{\pi}^{2}}^{\infty} \mathrm{d} s K(s) \operatorname{Im} \Pi(s), \\
K(s) & =\frac{\alpha^{2}}{3 \pi^{2} s} \hat{K}(s) ; \quad \hat{K}(s)=\int_{0}^{1} \mathrm{~d} x \frac{x^{2}(1-x)}{x^{2}+(1-x) s / m_{\mu}^{2}} .
\end{aligned}
$$

Here $\Pi$ is the hadronic part of the photon vacuum polarization function. Then, we can express $\operatorname{Im} \Pi$ in terms of the ratio of (lowest order) cross sections for $e^{+} e^{-}$annihilation into hadrons over annihilation into muons:

$$
\begin{gathered}
R(s)=\frac{\sigma^{(0)}\left(e^{+} e^{-} \rightarrow \text { hadrons }\right)}{\sigma^{(0)}\left(e^{+} e^{-} \rightarrow \mu^{+} \mu^{-}\right)}, \quad \sigma^{(0)}\left(e^{+} e^{-} \rightarrow \mu^{+} \mu^{-}\right) \equiv \frac{4 \pi \alpha^{2}}{3 s}: \\
\left.a_{\mu}^{(2)} \text { (Hadr. }\right)=\int_{4 m_{\pi}^{2}}^{\infty} \mathrm{d} s K(s) R(s) .
\end{gathered}
$$

Therefore, the situation is, in principle, simple: we take the experimental cross section for $e^{+} e^{-} \rightarrow$ hadrons, insert it into (2.1b) and the value for $a_{\mu}^{(2)}$ (Hadr.) will follow. In practice, however, things are more complex. The experimental numbers for the cross section $e^{+} e^{-}$into hadrons present (relatively) large errors and we have, therefore, interest to supplement these with more precise theoretical formulas whenever possible. As a matter of fact, by so doing we are able to diminish the error in the theoretical calculation almost by a factor of two. 
THE HADRONIC CONTRIBUTIONS TO THE ANOMALOUS MAGNETIC MOMENT OF THE MUON

2.1 The low energy region, $s \leq 0.8 \mathrm{GeV}^{2}$

In the region below $s=0.8 \mathrm{GeV}^{2}$, that we may call "rho region", experimental data have in fact improved substantially in the last years due to the more recent measurements, especially at Novosibirsk. ${ }^{[4,5]}$ Here the important contributions are those of the omega resonance (for which experimental data and the Gounnaris-Sakurai method may be used; see below and ref. 6) and the two-pion contribution, which is the one that may be made more precise using theory, and also the one that we will discuss in more detail because it provides the bulk of $a_{\mu}$ (Hadr.). Here one profits from the fact that the two pion contribution can be expressed in terms of the pion form factor, $F_{\pi}$,

$$
\operatorname{Im} \Pi_{2 \pi}(s)=\frac{1}{48 \pi}\left(1-\frac{4 m_{\pi}^{2}}{s}\right)^{3 / 2}\left|F_{\pi}(s)\right|^{2}
$$

and $F_{\pi}$ may be determined from fits to data on $e^{+} e^{-} \rightarrow 2 \pi$ and, using analyticity, also data from $\pi e \rightarrow \pi e$ scattering, i.e., at spacelike $s$. What is more, we may use (with due caution; see below) data on $\tau$ decay, $\tau^{ \pm} \rightarrow \nu \pi^{ \pm} \pi^{0}$, related to $F_{\pi}$ by isospin invariance.

We will for the moment work in the approximation of neglecting electroweak corrections to $F_{\pi}$; we will discuss this further in Subsect. 2.2. In this approximation, the properties of $F_{\pi}(s)$ that allow us an improved calculation are the following:

(i) $F_{\pi}(s)$ is an analytic function of $s$, with a cut from $4 m_{\pi}^{2}$ to infinity.

(ii) On the cut, the phase of $F_{\pi}(s)$ is, because of unitarity, identical to that of the Pwave in $\pi \pi$ scattering, $\delta_{1}^{1}(s)$, and this equality holds until the opening of the inelastic threshold at $s=t_{0}$ (Fermi-Watson final state interaction theorem).

(iii) For large $s, F_{\pi}(s) \simeq 1 / s$. Actually, one knows the coefficient of this behaviour, but we will not need it here.

(iv) $F_{\pi}(0)=1$.

The inelastic threshold occurs, rigorously speaking, at $s=16 \mathrm{~m}_{\pi}^{2}$. However, it is an experimental fact that inelasticity is negligible until the quasi-two body channels $\omega \pi, a_{1} \pi \ldots$ are open. In practice one can take

$$
t_{0} \simeq 1 \mathrm{GeV}^{2}
$$

and fix the best value for $t_{0}$ empirically. It will be $t_{0}=1.1 \mathrm{GeV}^{2}$, and the dependence of our results on $t_{0}$ is very slight.

The properties (i-iv) can be taken into account with the Omnès-Muskhelishvili method. ${ }^{1}$ We construct a function $J(s)$ with the proper phase and asymptotic behaviour by defining

$$
J(s)=\mathrm{e}^{1-\delta_{1}^{1}\left(t_{0}\right) / \pi}\left(1-\frac{s}{t_{0}}\right)^{\left[1-\delta_{1}^{1}\left(t_{0}\right) / \pi\right] t_{0} / s}\left(1-\frac{s}{t_{0}}\right)^{-1} \exp \left\{\frac{s}{\pi} \int_{4 m_{\pi}^{2}}^{t_{0}} \mathrm{~d} t \frac{\delta_{1}^{1}(t)}{t(t-s)}\right\} .
$$

\footnotetext{
$\overline{1}$ More details about the solution of the Omnès-Muskhelishvili equations can be found in the text of N. I. Muskhelishvili, Singular Integral Equations, Nordhoof, 1958, and, applied to our present case, in ref. 6 .
} 


\section{J. F. DE TROCÓNIZ AND F. J. YNDURÁIN}

We have written the dispersion relation with one subtraction to ensure that $J(0)=1$. The singular integral is understood to be calculated replacing $s \rightarrow s+\mathrm{i} \epsilon, \epsilon>0, \epsilon \rightarrow 0$. We then define the function $G$ by

$$
F_{\pi}(s)=G(s) J(s)
$$

and it follows from properties (i-ii) that $G(s)$ is analytic with only the exception of a cut from $t_{0}$ to infinity, as we have already extracted the correct phase below $s=t_{0}$.

We can apply the effective range theory to parametrize the phase $\delta_{1}^{1}$. According to this, the function

$$
\psi(t) \equiv \frac{2 k^{3}}{t^{1 / 2}} \cot \delta_{1}^{1}(t), \quad k=\frac{\sqrt{t-4 m_{\pi}^{2}}}{2}
$$

is analytic in the variable $t$ except for two cuts: a cut from $-\infty$ to 0 , and a cut from $t=t_{0}$ to $+\infty$. To profit from the analyticity properties of $\psi$ we will make a conformal transformation. We define

$$
w=\frac{\sqrt{t}-\sqrt{t_{0}-t}}{\sqrt{t}+\sqrt{t_{0}-t}}
$$

When $t$ runs the cuts, $w$ goes around the unit circle. We may therefore expand $\psi$ in a power series convergent inside the unit disc. In fact, because we know that the $\mathrm{P}$ wave resonates (which implies a zero of $\psi$ ) it is convenient to expand not $\psi$ itself, but the ratio $\psi(t) /\left(m_{\rho}^{2}-t\right) \equiv \hat{\psi}(t)$. Here $m_{\rho}$ is the mass of the rho resonance; so we write,

$$
\psi(t)=\left(m_{\rho}^{2}-t\right) \hat{\psi}(t)=\left(m_{\rho}^{2}-t\right)\left\{b_{0}+b_{1} w+\cdots\right\} .
$$

The P-wave, $I=1 \pi \pi$ scattering length, $a_{1}^{1}$, is related to $\psi$ by

$$
a_{1}^{1}=\frac{1}{m_{\pi} \psi\left(4 m_{\pi}^{2}\right)}
$$

experimentally, $a_{1}^{1} \simeq(0.038 \pm 0.003) m_{\pi}^{-3}$, a condition that may be incorporated into the fit. Note, however, that we do not assume the values of $m_{\rho}, \Gamma_{\rho}$. We only require that $\psi$ has a zero, and will let the fits fix its location and residue. It turns out that, to reproduce the width and scattering length, and to fit the pion form factor as well, only two $b_{0}, b_{1}$ are needed in $(2.4 \mathrm{~b})$.

We now turn to the function $G(s)$. This function is analytic except for a cut from $s=t_{0}$ to $+\infty$. The conformal transformation

$$
z=\frac{\frac{1}{2} \sqrt{t_{0}}-\sqrt{t_{0}-s}}{\frac{1}{2} \sqrt{t_{0}}+\sqrt{t_{0}-s}}
$$

maps this cut plane into the unit circle. So we may write the expansion,

$$
G(s)=1+c_{1}(z+1 / 3)+c_{2}\left(z^{2}-1 / 9\right)+\cdots,
$$

which takes into account the condition $G(0)=1$ order by order. We will only need two terms in the expansion, so we have $c_{1}, c_{2}$ as free parameters. This means that, altogether, we have the five parameters,

$$
m_{\rho}, b_{0}, b_{1}, c_{1}, c_{2}
$$


to fit 158 experimental points.

One can then use the formulas just discussed and fit the experimental data on $F_{\pi}(s)$, after we have taken into account the $\omega-\rho$ interference (which includes the $\omega \rightarrow 2 \pi$ piece). This we do with the Gounnaris-Sakurai method. We write

$$
F_{\pi}^{\text {all }}(s)=F_{\pi}^{\text {bare }}(s) \times \frac{1+\sigma \frac{M_{\omega}^{2}}{M_{\omega}^{2}-s}}{1+\sigma}, \quad M_{\omega}=m_{\omega}-\mathrm{i} \Gamma_{\omega} / 2
$$

where $F_{\pi}^{\text {bare }}$ is the form factor we would have in absence of $\omega-\rho$ interference and $m_{\omega}$ the (real) omega mass. We take the values of $m_{\omega}, \Gamma_{\omega}$ from the Particle Data Tables and find

$$
|\sigma|=(18 \pm 1) \times 10^{-4}, \quad \arg \sigma=12 \pm 3^{\circ} .
$$

The fit improves when using the 2002 Novosibirsk data; ${ }^{[4]}$ there is better agreement with the data of Barkov et al., ${ }^{[4]}$ in particular for the larger values of $s$. The parameters of the fit are, however, similar to what we found in ref. 6 using the old (1999) Novosibirsk data, as we shall see. It should be noted that, in these data, electromagnetic corrections have been extracted; we give the details of the procedure in Subsect. 2.2 below.

The result of the contribution to the hadronic part of the muon anomaly is now, fitting the $e^{+} e^{-}$annihilation data, ${ }^{[4,5]}$

$$
10^{11} \times a_{\mu}^{(2)}\left(s \leq 0.8 \mathrm{GeV}^{2}\right)=4707 \pm 21 ; \quad \chi^{2} / \text { d.o.f. }=91 /(114-7) .
$$

The fact that the $\chi^{2} /$ d.o.f. is substantially smaller than unity means that there is some room for displacement of the central value given in (2.7).

The result in (2.7) number may be improved, which we will do in two steps. First of all, we remark that, because our formulas for $F_{\pi}(s)$ are valid for spacelike as well as timelike $s$, we can use information not only from $e^{+} e^{-}$annihilation, ${ }^{[4,5]}$ but also from $\pi e$ scattering, ${ }^{[7]}$ which gives $F_{\pi}(s)$ for negative $s$. We record the results of two recent evaluations, made using this method. We have our evaluation here,

$$
10^{11} \times a_{\mu}^{(2)}\left(s \leq 0.8 \mathrm{GeV}^{2}\right)=\left\{\begin{array}{l}
4750 \pm 19 \quad[\mathrm{TY} ; \text { fixed norm. }] ; \frac{\chi^{2}}{\text { d.o.f. }}=\frac{172}{159-7} \\
4715 \pm 20 \pm 25 \quad[\mathrm{TY} ; \text { float. norm. }] ; \frac{\chi^{2}}{\text { d.o.f. }}=\frac{134}{161-10} .
\end{array}\right.
$$

The errors in the first number here do not include systematic errors in $e \pi$ scattering, which is the reason for the largish $\chi^{2} /$ d.o.f. In the second set, the first error is the statistical error, the second the systematic one. We take into account the systematic errors, both in $e^{+} e^{-}$ data $^{[4]}$ and in the $e \pi$ data $^{[7]}$ as in ref. 6 , by including a factor $1+\epsilon$ in the normalization of each set of data, and allowing $\epsilon$ to float. We find

$$
\begin{aligned}
& \epsilon_{e \pi[\text { ref. } 7]}=(1.3 \pm 0.2) \%, \quad \epsilon_{e^{+} e^{-}[\text {Akhmetshin, ref.4] }}=(-0.4 \pm 0.6) \%, \\
& \epsilon_{e^{+} e^{-}[\text {OLYA; Barkov, ref.4] }}=(0.5 \pm 0.9) \% ;
\end{aligned}
$$


J. F. DE TROCÓNIZ AND F. J. YNDURÁIN

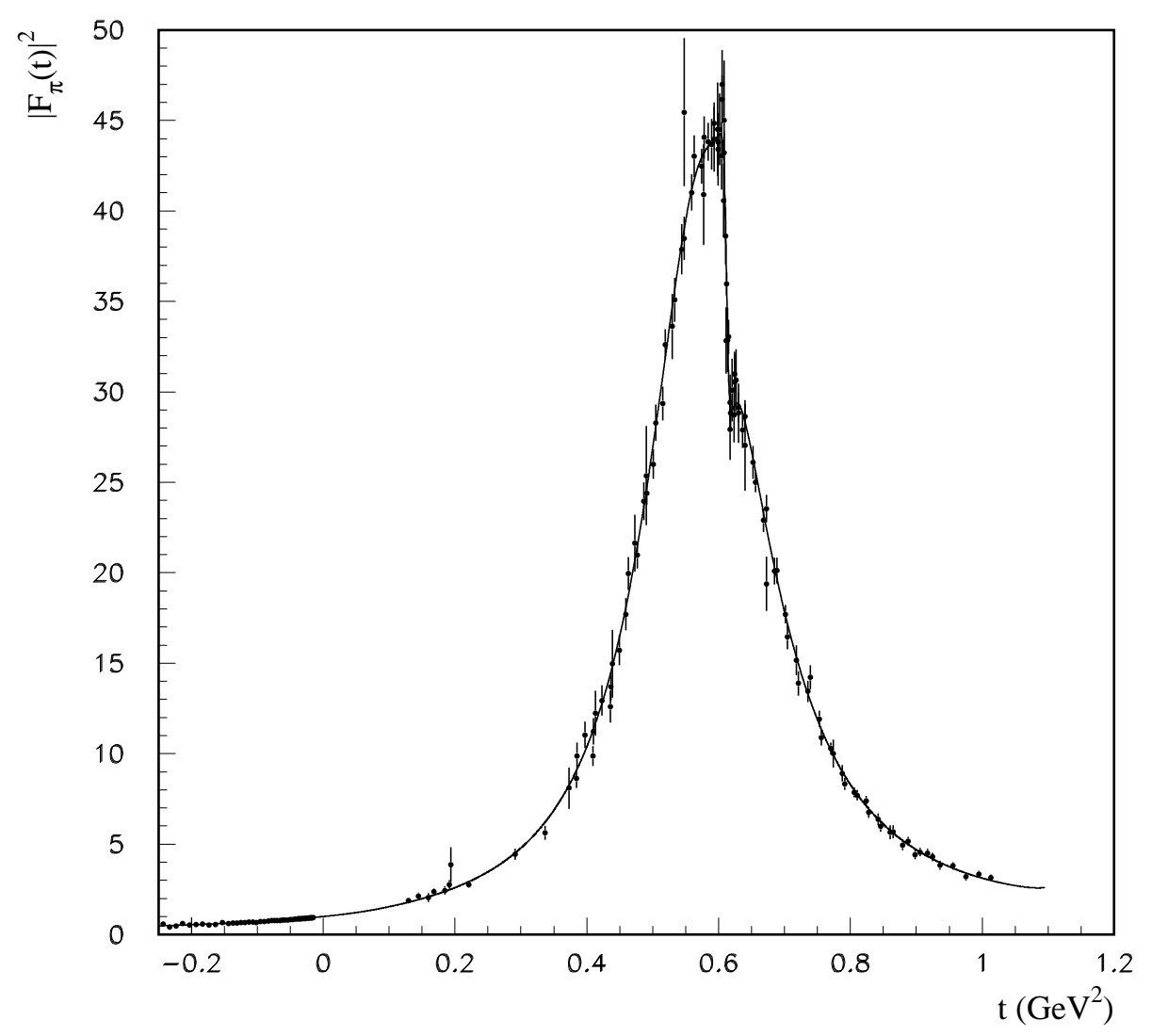

Figure 2. The fit to the pion form factor data in the timelike and spacelike regions; the more recent (2002) Novosibirsk data are included.

the fit is depicted in Fig. 2. The only $\epsilon$ that is not compatible with zero is $\epsilon_{e \pi}$. In fact, we left its value arbitrary; that the result we found is consistent with the value quoted in ref. 7 itself, $\epsilon_{e \pi} \simeq 1 \%$, is a nontrivial test of the quality of data in this reference (in particular, of their estimate of systematic errors) and of the consistency of our fitting procedure.

Then, we have a recent result by Colangelo and collaborators, ${ }^{[8]}$

$$
\left[e^{+} e^{-}, \pi e\right]: \quad 10^{11} \times a_{\mu}^{(2)}\left(s \leq 0.8 \mathrm{GeV}^{2}\right)=4679 \pm 30 \quad \text { [Colangelo, ref. 8] } .
$$

The evaluation of Colangelo and collaborators, Eq. (2.9), includes the four-pion cut, and imposes the $\mathrm{P}$ wave phase shift as given by chiral-dispersive evaluations. ${ }^{[9]}$ This last feature, however, makes the Colangelo result vulnerable to possible defects of the chiraldispersive evaluation, such as those discussed in ref. 10. 
A way to improve the precision of $(2.7)$ is to include information on $\tau$ decay ${ }^{[11]}$ in the fit. We consider the weak vector current correlator, related to $\tau$ decay, and expand it as

$$
\begin{aligned}
\Pi_{\mu \nu}^{V} & =\mathrm{i} \int \mathrm{d}^{4} x \mathrm{e}^{\mathrm{i} p \cdot x}\left\langle 0\left|\mathrm{~T} V_{\mu}^{+}(x) V_{\nu}(0)\right| 0\right\rangle=\left(-p^{2} g_{\mu \nu}+p_{\mu} p_{\nu}\right) \Pi^{V}(t)+p_{\mu} p_{\nu} \Pi^{S}(t) ; \\
v_{1} & \equiv 2 \pi \operatorname{Im} \Pi^{V}, \quad t=p^{2} .
\end{aligned}
$$

We can then write

$$
v_{1}=\frac{1}{12}\left\{\left[1-\frac{\left(m_{\pi^{+}}-m_{\pi^{0}}\right)^{2}}{t}\right]\left[1-\frac{\left(m_{\pi^{+}}+m_{\pi^{0}}\right)^{2}}{t}\right]\right\}^{3 / 2}\left|F_{\pi}^{(\tau)}(t)\right|^{2} .
$$

To compare with the experimentally measured quantity, which involves all of $\operatorname{Im} \Pi_{\mu \nu}^{V}$, we have to neglect the scalar component $\Pi^{S}$, which is proportional to $\left(m_{d}-m_{u}\right)^{2}$, and thus very small. Moreover, $F_{\pi}^{(\tau)}$ only equals $F_{\pi}$ in the limit of exact isospin invariance. We have also complications due to radiative corrections, that we will discuss in Subsect. 2.3.

One can take into account isospin breaking effects, at least partially, by realizing that the mass and widths of the $\rho^{0}$ and $\rho^{ \pm}$can be different. ${ }^{[6]}$ One finds,

$$
\begin{aligned}
& {\left[e^{+} e^{-}, \pi e, \tau\right]:} \\
& 10^{11} \times a_{\mu}^{(2)}\left(s \leq 0.8 \mathrm{GeV}^{2}\right)=\left\{\begin{array}{l}
4793 \pm 17 \text { (St.), } \quad \frac{\chi^{2}}{\text { d.o.f. }}=\frac{283}{241-9} ;[\mathrm{TY}] \\
4798 \pm 17 \text { (St.) } \pm 25 \text { (Sys.), } \frac{\chi^{2}}{\text { d.o.f. }}=\frac{245}{244-13} ;[\mathrm{TY}, \text { Sys. }]
\end{array}\right.
\end{aligned}
$$

depending on whether or not one takes into account systematic normalization errors. We unify the normalization of the tau decay data taking into account the relevant branching ratios, as given in the Particle Data Tables. ${ }^{2}$

In the second set of numbers in (2.11), we have, as in (2.8), taken into account systematic errors by allowing floating normalization by a factor $1+\epsilon$ of the various data sets. We find,

$$
\begin{aligned}
& \epsilon_{e \pi[\text { ref. } 7]}=(1.0 \pm 0.2) \% \quad[1 \%], \quad \epsilon_{e^{+} e^{-} \text {[Akhmetshin, ref.4] }}=(0.4 \pm 0.5) \% \quad[0.6 \%], \\
& \epsilon_{\tau}=(-1.4 \pm 0.5) \% \quad[0.7 \%], \quad \epsilon_{e^{+} e^{-}[\text {OLYA; Barkov, ref. } 4]}=(2.0 \pm 0.8) \% \quad[4 \%] .
\end{aligned}
$$

In square brackets we give the estimate of the normalization errors as given by the experimental groups themselves, except for $\tau$ decay, where the number $0.7 \%$ is taken from the Particle Data Tables. The only case where the value we find for $\epsilon$ exceeds the expectations is for $\tau$ decay data, although the difference is very small, $0.7 \pm 0.5 \%$. We will discuss this again in Sect. 2.3.

The best number in (2.11a), of course, is the second, $4798 \pm 31$. The fact that the $\chi^{2} /$ d.o.f. is still a bit larger than unity can be traced partly to the size of $\epsilon_{\tau}$, that we

${ }^{2}$ When quoting the PDT we refer to the 2002 edition, K. Hagiwara et al., Phys. Rev. D66, 010001 (2002). 
discuss in Subsect. 2.3, and partly to a discrepancy between the tau decay data of OPAL and of ALEPH and CLEO. This is seen very clearly if we give the individual values for the ratio of $\chi^{2}$ to number of experimental points of the various sets of data, which we do for the second fit in (2.11a), i.e., including systematic errors:

$$
\begin{array}{ccc}
e \pi \quad \text { [NA7, ref. 7] : } & 42 / 45 \\
e^{+} e^{-} \text {[ref. 4] : } & 108 / 113 \\
\tau \text { decay [Aleph, ref. 11] : } & 19 / 21 \\
\tau \text { decay [Cleo, ref. 11] : } & 32 / 30 \\
\tau \text { decay [Opal, ref. 11] : } & 40 / 31 .
\end{array}
$$

A remarkable feature of (2.11c) is that it shows that including information from tau decay in the fit does not spoil the quality of the fit of the pure $e^{+} e^{-}$data.

\subsection{Radiative corrections for the $e^{+} e^{-}$case}

We next devote a few words to discuss electromagnetic radiative corrections, a subject of crucial importance given the precision of the more recent data, but not always very clear in the existing literature.

There are, in fact, two separate questions here. First, we have the radiative corrections to the hadronic part of the photon vacuum polarization, which affect Fig. 1 by adding photon corrections (depicted below, in Fig. 6). These give corrections to $a_{\mu}$ of order $\alpha^{3}$ and will be considered later, in Sect. 3. Secondly, we have the matter of the radiative corrections that have to be taken into account when extracting the pion form factor from experimental data. These come about for the following reason: the form factor that verifies the analyticity and unitarity properties necessary to carry out our analysis in Subsect. 2.1 is defined by

$$
\left.\left\langle p\left|J_{\mu}(0)\right| p^{\prime}\right\rangle\right|_{\text {electroweak int. } \equiv 0}=(2 \pi)^{-3}\left(p-p^{\prime}\right)_{\mu} F_{\pi}\left(\left(p-p^{\prime}\right)^{2}\right),
$$

i.e., only strong interactions are taken into account for the expectation value of the electromagnetic current, $J_{\mu}$.

This quantity, $F_{\pi}$, has thus to be extracted from the experimentally measured cross sections for $e^{+} e^{-} \rightarrow \pi^{+} \pi^{-}$, which include all sorts of radiative corrections. To first (relative) order in $\alpha$, these are the following: I) Corrections to the $e^{+} e^{-} \gamma$ vertex, or photon radiation by the incoming $e^{+}, e^{-}$. These are pure QED effects readily calculated and taken into account as a matter of course in experimental analyses. II) Vacuum polarization corrections to the photon propagator. These are known in terms of $\operatorname{Im} \Pi$, and are also be subtracted easily. They are explicitly taken into account in the second paper in ref. 4 . III) Corrections to the $\pi^{+} \pi^{-} \gamma$ vertex, or photon radiation by the outgoing pions (Fig. 3). We now say a few words about the last.

Radiation of hard photons by the outgoing pions, as in Fig. 3B, is excluded by the experimental cuts applied when analyzing $e^{+} e^{-} \rightarrow \pi^{+} \pi^{-}$scattering, which require the angle between the momenta of $\pi^{+}, \pi^{-}$to be close to $180^{\circ}$. One is thus left with the soft photon radiation and vertex correction shown in Fig. 3A. They can be calculated under the assumption that one can factorize the pion form factor and, given the actual values of 


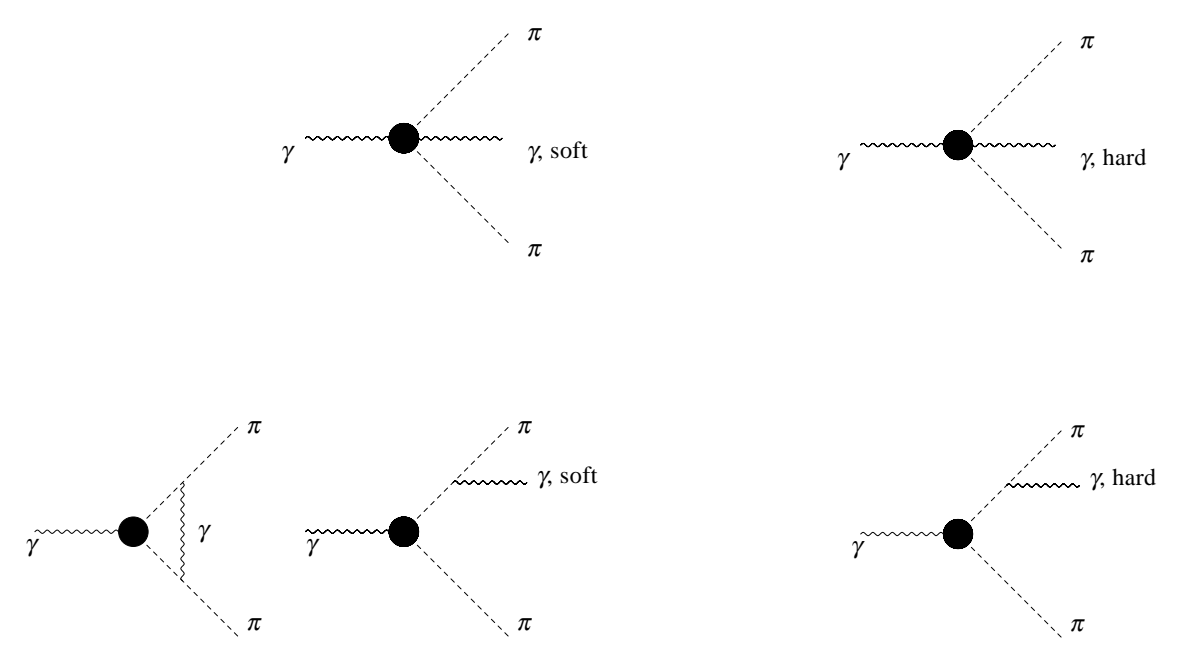

(A)

FIgURE 3. Diagrams subtracted for evaluating the pion form factor contribution in $a_{\mu}^{(2)}\left(s \leq 0.8 \mathrm{GeV}^{2}\right)$, but included in the $O\left(\alpha^{3}\right)$ contribution to $a_{\mu}$ (Hadr.). The blob represents the pion form factor, to zero order in electroweak interactions, as defined in Eq. (2.12).

the experimental cuts applied to the momenta of the $\pi^{+}, \pi^{-}$, this correction turns out to be very small. In this approximation, the corrections have been evaluated long ago and are, fortunately, explicitly given and extracted in the 2002 version of the Novosibirsk data (ref. 4).

From this analysis it follows that, for the $e^{+} e^{-} \rightarrow \pi^{+} \pi^{-}$case, one can subtract all corrections and really obtain $F_{\pi}$, as defined in Eq. (2.12), from data with an error that is only of order $\alpha^{2}$. This is the quantity to which we can apply our theoretical analysis, as we did at the beginning of the present Section.

A problem with the evaluation of the radiative corrections here, however, is that, as noted, one is using a model with elementary pions, in which the form factor is included by hand (factorized). This may cause errors (for example, due to rescattering of the pions or dependence of the form factor on the off-shell pion mass), whose size (likely small, since the correction itself is small) we will estimate when discussing the case of tau decay in next Subsection.

We should here note that the radiative corrections to the $\pi^{+} \pi^{-} \gamma$ vertex, or photon radiation by the outgoing pions will have to be considered again when we consider 
contributions to $a_{\mu}$ due to photon vacuum polarization of $O\left(\alpha^{3}\right)$. This we will do in Sect. 3 .

\subsection{Comment on combining results using $e^{+} e^{-} \rightarrow \pi \pi$ and $\tau \rightarrow \nu \pi \pi$, and on radiative corrections for $\tau$ decay}

In some recent papers much ado is made about the difference in the pion form factor, in the region near and above the rho resonance, depending whether it its extracted from $e^{+} e^{-} \rightarrow \pi \pi$ or from $\tau \rightarrow \nu \pi \pi$. In fact, this difference is expected. To begin with, $|F(s)|^{2}$ grows almost by a factor 50 around the rho. ${ }^{3}$ Thus, even a small difference between the masses and widths of $\rho^{ \pm}$and $\rho^{0}$ will imply a large difference in the form factors. This matter was studied carefully in ref. 6 , which discussion we summarize and update now.

The values for the anomaly in the rho region that one obtains depending what one fits, is, with only statistical errors,

$$
10^{11} \times a_{\mu}^{(2)}\left(s \leq 0.8 \mathrm{GeV}^{2}\right)= \begin{cases}4707 \pm 21 & {\left[\text { from } e^{+} e^{-} \rightarrow \pi \pi \text { only }\right]} \\ 4820 \pm 11 & {[\text { from } \tau \rightarrow \nu \pi \pi \text { only }]}\end{cases}
$$

For the tau, we have $\chi^{2} /$ d.o.f. $=87 /(83-5)$. The two numbers are well outside each other error bars and it is, therefore, dangerous to combine them in a direct manner. What, however, one can do, is to fit simultaneously $e^{+} e^{-} \rightarrow \pi \pi$ and $\tau \rightarrow \nu \pi \pi$ data allowing for different values of the parameters $m_{\rho}, b_{0}, b_{1}$, (and therefore, also different widths ${ }^{4}$ ) but with the same Omnès-Muskhelishvili function $G(s)$ in Eq. (2.3b). As discussed in ref. 6, we expect isospin breaking effects to be small for $G(s)$ since its imaginary part is different from zero only for $s>1.1 \mathrm{GeV}^{2}$.

Of course, when calculating $a_{\mu}\left(s \leq 0.8 \mathrm{GeV}^{2}\right)$ one uses the parameters $m_{\rho}, b_{0}, b_{1}$ determined from the fit to $e^{+} e^{-} \rightarrow \pi \pi$, even if we use tau decay data to help fix $G$ : we should perhaps emphasize that the result reported in (2.11) is not an average of $e^{+} e^{-}$and $\tau$ results, but an evaluation of $e^{+} e^{-} \rightarrow \pi \pi$, using information on $G(s)$ from tau decay.

This use of different masses and widths for $\rho^{ \pm}, \rho^{0}$ is not sufficient to remove the discrepancies between the form factors obtained from $e^{+} e^{-}$and $\tau$ decay. A reason for at least part of the remaining discrepancy is that the experimental number given in ref. 11 for $\tau$ decay includes also the radiative decay; that is to say, one does not measure the quantity $\Gamma(\tau \rightarrow \nu \pi \pi)$ but, in fact, $\Gamma(\tau \rightarrow \nu \pi \pi)+\Gamma(\tau \rightarrow \nu \pi \pi+\gamma)+$ higher orders. So we should discuss radiative corrections also for tau decay.

First of all, we must make more explicit what we used in our fits. We take the experimental numbers for the decay $\tau \rightarrow \nu \pi \pi$ including an eventual photon. The corresponding width we denote by $\Gamma_{\text {exp. }}\left(\tau \rightarrow \nu \pi^{ \pm} \pi^{0}(\gamma)\right)$, and we divide this by the experimental decay rate for $\tau \rightarrow \nu_{\tau} e \nu_{e}$, plus eventual gammas, $\Gamma_{\text {exp. }}\left(\tau \rightarrow \nu_{\tau} e \nu_{e}(\gamma)\right)$. However, and as in the case of $e^{+} e^{-}$annihilation, we would like to have the quantity $F_{\pi}^{(\tau)}$ with electroweak

$\overline{3}$ There is also a significant difference between data from $e^{+} e^{-}$and tau decay for $0.8 \mathrm{GeV}^{2}<$ $s<1.1 \mathrm{GeV}^{2}$ which, however, affects very little the result for $a_{\mu}\left(s \leq 0.8 \mathrm{GeV}^{2}\right)$.

${ }^{4}$ We take, however, equal $\mathrm{P}$ wave scattering lengths for $\pi^{+} \pi^{-}$and $\pi^{0} \pi^{ \pm}$. We have checked that the influence of this is negligible. 

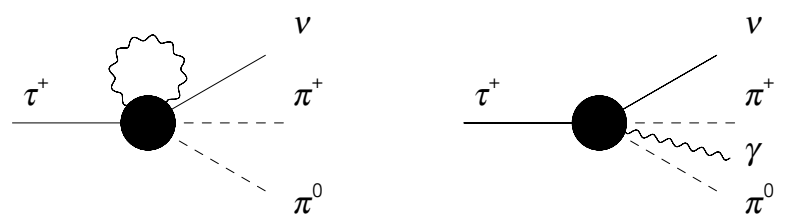

FIGURE 4. Radiative corrections to $\tau \rightarrow \nu \pi \pi$ decay.

interactions set to zero. Therefore, we should correct the ratio

$$
\frac{\Gamma_{\text {exp. }}\left(\tau \rightarrow \nu \pi^{ \pm} \pi^{0}(\gamma)\right)}{\Gamma_{\text {exp. }}\left(\tau \rightarrow \nu_{\tau} e \nu_{e}(\gamma)\right)}
$$

to obtain the quantity

$$
\frac{\Gamma^{(0)}\left(\tau \rightarrow \nu \pi^{ \pm} \pi^{0}\right)}{\Gamma^{(0)}\left(\tau \rightarrow \nu_{\tau} e \nu_{e}\right)}
$$

where the $\Gamma^{(0)}$ are evaluated to lowest order in electroweak interactions.

The corrections necessary to do this are as follows. We first have radiative corrections to the leptonic width, that give

$$
\Gamma_{\exp .}\left(\tau \rightarrow \nu_{\tau} e \nu_{e}(\gamma)\right)=\left(1+\delta_{e}\right) \Gamma^{(0)}\left(\tau \rightarrow \nu \pi^{ \pm} \pi^{0}\right), \quad \delta_{e} \simeq\left(\frac{25}{4}-\pi^{2}\right) \frac{\alpha}{2 \pi} \simeq-0.004
$$

This correction was incorporated in the analysis of Subsect. 2.1.

Then we have the corrections to the hadronic width. We here have corrections similar to those in Fig. 3B; and diagrams similar to those in Fig. 3A: see Fig. 4. We start with the first, that is to say, the sum of loop corrections (plus radiation of a soft photon). As far as we know, this has not been calculated exactly. However, we may expect this to be dominated by the short distance piece, since this last contains a large logarithm, $\log M_{Z} / m_{\tau}$. This gives the correction

$$
1+\frac{2 \alpha}{\pi} \log \frac{M_{Z}}{m_{\tau}} \simeq 1.019
$$

as has been known from a long time. ${ }^{[12]}$ This piece we have also extracted in our analysis above. After so doing, there still remains a piece of the loop correction and a correction due to soft photon radiation. Since we have extracted the large $\log M_{Z} / m_{\tau}$ piece, we expect this to be comparable to the like piece in $e^{+} e^{-} \rightarrow \pi \pi$ case, and thus small. 
J. F. DE TROCÓNIZ AND F. J. YNDURÁIN

It remains to correct for the ratio

$$
1+\delta_{\gamma} \equiv \frac{\Gamma(\tau \rightarrow \nu \pi \pi)+\Gamma(\tau \rightarrow \nu \pi \pi+\gamma)}{\Gamma^{(0)}(\tau \rightarrow \nu \pi \pi)},
$$

with $\gamma$ a hard photon, i.e., a photon with energy larger than a given $E_{0}$. This, again, is not known, but one can approximate it by the infrared logarithmic piece, which gives an average correction

$$
\delta_{\gamma} \simeq \frac{\alpha}{\pi} \log \frac{m_{\tau}-\bar{M}_{\pi \pi}}{E_{0}} \simeq 0.008,
$$

the last for $20 \mathrm{MeV} \leq E_{0} \leq 80 \mathrm{MeV}$, and we have neglected terms of $O\left(m_{\pi} / m_{\tau}\right) . \bar{M}_{\pi \pi}$ is the average invariant mass of the two pions, that we take equal to $m_{\rho}$. Since this correction plus the remainder (after extracting the logarithm) of the loop correction are not known exactly, we have not included the correction (2.16) in our evaluations above: (2.16) will be part of the normalization factor $\epsilon_{\tau}$.

When we allowed variations of the normalization for tau decay data, ${ }^{[11]}$ multiplying their numbers by $1+\epsilon$, and letting $\epsilon$ float, we found the excellent fit reported in (2.11) with $\epsilon_{\tau}=-1.4 \%$. It unfortunately is not possible to understand all of this as due only to the neglect of radiative corrections; we have verified that, including $\delta_{\gamma}$ as given in (2.16) in the fit only changes $\epsilon_{\tau}$ to $-1.2 \%$. We have to admit that there is a residual discrepancy with the value for the error in normalization, $\left|\epsilon_{\tau}\right|=0.7 \%$, given in the Particle Data Tables; but, the difference is small and, indeed, we get a fit to all experimental data with a $\chi^{2}$ per experimental point which is essentially unity.

In spite of this, it is clear that here we have not fully determined radiative corrections; only the $\operatorname{logarithms} \log M_{Z}, \log E_{0}$ are exact in Eqs. $(2.14,16)$. Moreover, the function $G(s)$ will not be exactly invariant under isospin and, finally, a correction due to the fact that $m_{u} \neq m_{d}$, although likely very small, also exists beyond the rho. We must thus conclude that our partial ignorance of isospin violations, very likely dominated by radiative corrections, implies a possible shift of the central value of the anomaly. A conservative estimate for these effects would be the difference between the two values obtained leaving the $\tau$ decay normalization fixed, and the same allowing it to float. This gives the number

$$
\Delta_{\text {rad }}\left[10^{11} \times a_{\mu}^{(2)}\left(s \leq 0.8 \mathrm{GeV}^{2}\right)\right] \simeq 10 .
$$

We will accept the same error for the process $e^{+} e^{-} \rightarrow \pi \pi$, although it is probably smaller here.

\subsection{The region with $s>0.8 \mathrm{GeV}^{2}$}

For low energies (say, $s<2 \mathrm{GeV}^{2}$ ), and near quark thresholds, there is no alternative to using experimental data. ${ }^{[12]}$ Between $0.8 \mathrm{GeV}^{2}$ and $1.2 \mathrm{GeV}^{2}$ we fit the experimental data $^{[4]}$ for the $\pi^{+} \pi^{-}$channel; its contribution is $(229 \pm 3 \pm 3) \times 10^{-11}$. For other final states $(\bar{K} K, 3 \pi, 4 \pi, \ldots)$ we use the $e^{+} e^{-}$data of refs. 5 and 13 , with the methods of ref. 6. For higher energies, $s>2 \mathrm{GeV}^{2}$, and away from quark thresholds, we can use QCD formulas, ${ }^{[14]}$ taking into account the more recent values of the masses of the quarks as well 


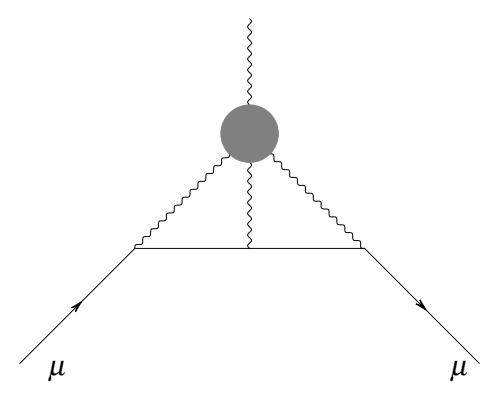

FiguRe 5. A typical diagram for the hadronic light by light contributions to the muon magnetic moment.

as the strong coupling, $\alpha_{s}$. For the QCD calculations we take the following approximation: for $n_{f}$ massless quark flavours, with charges $Q_{f}$, we write

$$
\begin{aligned}
R^{(0)}(s) & =3 \sum_{f} Q_{f}^{2}\left\{1+\frac{\alpha_{s}}{\pi}+\left(1.986-0.115 n_{f}\right)\left(\frac{\alpha_{s}}{\pi}\right)^{2}\right. \\
& \left.+\left[-6.64-1.20 n_{f}-0.005 n_{f}^{2}-1.240 \frac{\left(\sum_{f} Q_{f}\right)^{2}}{3\left(\sum_{f} Q_{f}^{2}\right)}\right]\left(\frac{\alpha_{s}}{\pi}\right)^{3}\right\} .
\end{aligned}
$$

To this one adds mass and nonperturbative corrections. We take into account the $O\left(\mathrm{~m}^{2}\right)$ effect for $s, c, b$ quarks with running masses $\bar{m}_{i}(s)$, which correct $R^{(0)}$ by the amount

$$
-3 \sum_{i=s, c, b} Q_{i}^{2} \bar{m}_{i}^{2}(s)\left\{6+28 \frac{\alpha_{s}}{\pi}+\left(294.8-12.3 n_{f}\right)\left(\frac{\alpha_{s}}{\pi}\right)^{2}\right\} s^{-1}
$$

The details may be seen in refs. 6, 15 .

Adding all the contributions, one has

$$
10^{11} \times a_{\mu}^{(2)}\left(s \geq 0.8 \mathrm{GeV}^{2}\right)=2134 \pm 35 .
$$

This number is almost the same that may be found in ref. 6, using the 1999 Novosibirsk data; including the new data has very little influence in this region.

\section{The hadronic contributions to $a_{\mu}$. II: the $O\left(\alpha^{3}\right)$ pieces}

A contribution in a class by itself is the hadronic light by light one, that we label " $\ell \times \ell$ ". So we split

$$
a\left(\text { Hadronic, } O\left(\alpha^{3}\right)\right)=a\left(\text { 'One blob' hadronic, } O\left(\alpha^{3}\right)\right)+a(\ell \times \ell) \text {. }
$$




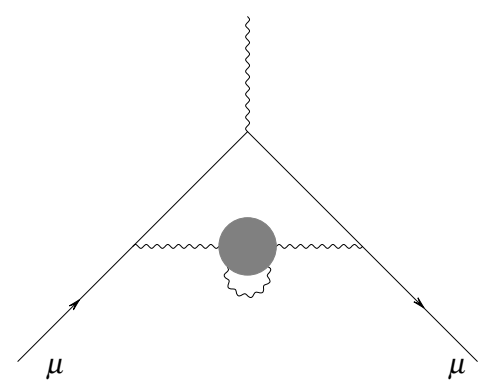

Figure 6. The $O\left(\alpha^{3}\right)$ hadronic correction $a($ h.v.p., $\gamma)$.

We will start by considering the last, given diagrammatically by graphs like that of Fig. 5. This can be evaluated only using models. One can make a chiral model calculation (essentially, replacing the hadronic blob by the lightest hadronic state, a $\pi^{0}$ ), or one can use a constituent quark model in which we replace the blob in Fig. 5 by a quark loop. For the chiral model calculation we have to introduce a cut-off, since the $\pi^{0}$ contribution diverges for large virtuality of the photon lines. The result depends on the cut-off (for the chiral calculation) or on the constituent mass chosen for the quarks. After the correction of a sign error in the evaluations of ref. 16 (see ref. 17) we find

$$
10^{11} \times a(\ell \times \ell)=86 \pm 25 \quad \text { [Chiral calculation]. }
$$

Earlier calculations with the $\pi^{0}$ model, using VMD to cure its divergence, gave (HKS, ref. 16)

$$
10^{11} \times a(\ell \times \ell)=52 \pm 20 \quad\left[\pi^{0} \text { pole }(\mathrm{HKS})\right] .
$$

One could also take the estimate of the $\pi^{0}$ pole from Hayakawa, Kinoshita and Sanda ${ }^{[16]}$ and add the constituent quark loop, in which case we get

$$
10^{11} \times a(\ell \times \ell)=98 \pm 22 \quad \text { [Quark const. model+ pion pole] } .
$$

One expects the $\pi^{0}$-dominated calculation to be valid for small values of the virtual photon momenta, and the constituent model to hold for large values of the same. Thus, almost half of the contribution to $a(\ell \times \ell)$ in the chiral calculation comes from a region of momenta above $0.5 \mathrm{GeV}$, where the chiral perturbation theory starts to fail, while for this range of energies, and at least for the imaginary part of (diagonal) light by light scattering, the quark model reproduces reasonably well the experimental data, as measured in photonphoton scattering. In view of this, we will take here the figure

$$
10^{11} \times a(\ell \times \ell)=92 \pm 30,
$$

but will refrain from combining this error with the others.

We next turn to the $a$ ('One blob' hadronic, $O\left(\alpha^{3}\right)$ ) corrections, which are obtained by attaching a photon or fermion loop to the various lines in Fig. 1. They can be 
further split into two pieces: the piece where both ends of the photon line are attached to the hadron blob, $a$ (h.v.p., $\gamma$ ), hadronic vacuum polarization corrections, shown in Fig. 6, and the rest. So we write,

$$
\left.a\left(\text { 'One blob' hadronic, } O\left(\alpha^{3}\right)\right)=a \text { (h.v.p., } \gamma\right)+a(\text { 'One blob' hadronic, rest). }
$$

The last can be evaluated ${ }^{[18]}$ in terms of the hadronic contributions to the photon vacuum polarization, finding

$$
10^{11} \times a(\text { 'One blob' hadronic, rest })=-101 \pm 6 .
$$

This result has been checked independently recently, by the Marseilles group (S. Friot, D. Greynat; E. de Rafael, private communication) and in ref. 21.

The only contribution that requires further discussion is that depicted in Fig. 6, $a$ (h.v.p., $\gamma$ ). In principle, this contribution can be evaluated straightforwardly by a generalization of the method used to evaluate the $O\left(\alpha^{2}\right)$ contributions. We can write

$$
a^{(2)}(\text { Hadr. })+a(\text { h.v.p., } \gamma)=\int_{4 m_{\pi}^{2}}^{\infty} \mathrm{d} t K(t) R^{(2)}(t)
$$

where

$$
R^{(2)}(t)=\frac{\sigma^{(0)}\left(e^{+} e^{-} \rightarrow \text { hadrons }\right)+\sigma^{(2)}\left(e^{+} e^{-} \rightarrow \text { hadrons }\right)+\sigma^{(0)}\left(e^{+} e^{-} \rightarrow \text { hadrons; } \gamma\right)}{\sigma^{(0)}\left(e^{+} e^{-} \rightarrow \mu^{+} \mu^{-}\right)} .
$$

The notation means that we evaluate the hadron annihilation cross section to second order in $\alpha$, and we add to it the first order annihilation into hadrons plus a photon. For energy large enough this can be calculated with the parton model, which leads to a (very small) correction, $(2 \pm 1) \times 10^{-11}$.

Then comes the contribution of small momenta. We start by discussing the process involving two pions. We calculate the corresponding piece by adding the contribution of the diagrams in Fig. 3, as given in the 2002 paper by Akhmetshin et al. ${ }^{[4]}$ In this way, we find

$$
10^{11} \times a\left(\text { h.v.p., } \pi^{+} \pi^{-} \gamma\right)=47.6 \pm 0.3 .
$$

The number is very close to that obtained in ref. $6(46 \pm 9)$ but the errors have decreased drastically. ${ }^{5} \mathrm{~A}$ similar analysis ought to be made, in principle, for other radiative intermediate states like $3 \pi+\gamma$ and $\bar{K} K+\gamma$, which can be estimated in terms of the corresponding decays of the $\omega$ and $\phi$, but they give a contribution below the $10^{-11}$ level and we neglect them. The contribution from $\pi^{0} \pi^{0} \gamma,(2.0 \pm 0.3) \times 10^{-11}$, is taken from ref. 7 .

The lowest energy contributions to $\sigma^{(0)}\left(e^{+} e^{-} \rightarrow\right.$ hadrons; $\left.\gamma\right)$ are those of the intermediate states $\pi^{0} \gamma$ and $\eta \gamma$, Fig. 7. These contributions were evaluated in detail in ref. 6 ; we have

$$
\begin{gathered}
10^{11} \times a\left(\text { h.v.p., } \pi^{0} \gamma\right)=37 \pm 3 \\
10^{11} \times a(\text { h.v.p., } \eta \gamma)=6 \pm 2
\end{gathered}
$$

\footnotetext{
${ }^{5}$ In ref. 6 only the radiation of hard photons was included, evaluated using the results of ref. 19. The fact that this is so similar to the full result justifies the (expected) smallness of soft photon plus vertex correction.
} 


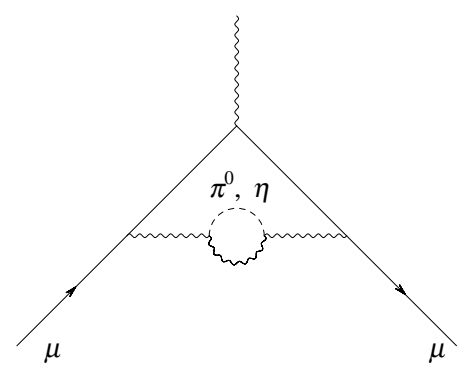

Figure 7. The $\pi^{0} \gamma$, $\eta \gamma$ contributions to $a$ (h.v.p., $\gamma)$.

Collecting all of this, we get the total effect of the states hadrons $+\gamma$, including the loop correction,

$$
10^{11} \times a(\text { h.v.p., } \gamma)=95 \pm 6 .
$$

\section{Byproducts}

4.1 The electromagnetic coupling on the $Z, \bar{\alpha}_{\text {Q.E.D. }}\left(M_{Z}^{2}\right)$

With a simple change of integration kernel the previous analysis can be extended to evaluate the hadronic contribution to the QED running coupling, $\bar{\alpha}_{\text {Q.E.D. }}(t)$, in particular on the $Z$ particle, $t=M_{Z}^{2}$; this is an important quantity that enters into precision evaluations of electroweak observables. By using a dispersion relation one can write this hadronic contribution at energy squared $t$ as

$$
\Delta_{\text {had }} \alpha(t)=-\frac{t \alpha}{3 \pi} \int_{4 m_{\pi}^{2}}^{\infty} \mathrm{d} s \frac{R(s)}{s(s-t)}
$$

where $R$ is as in (2.1b) and the integral has to be understood as a principal part integral. Therefore, we can carry over all the work from the previous sections as in ref. 20, with the simple replacement

$$
K(s) \rightarrow-\frac{t \alpha}{3 \pi} \frac{1}{s(s-t)}
$$

We find, to next to leading order in $\alpha$,

$$
10^{5} \times \Delta_{\text {had }} \alpha\left(M_{Z}^{2}\right)=2742 \pm 12
$$

or, excluding the top quark contribution,

$$
\begin{gathered}
10^{5} \times \Delta_{\text {had }} \alpha^{(5)}\left(M_{Z}^{2}\right)=2749 \pm 12 . \\
-16-
\end{gathered}
$$


Adding the known pure QED corrections, the running QED coupling, in the momentum scheme, is

$$
\bar{\alpha}_{\text {Q.E.D. }}\left(M_{Z}^{2}\right)=\frac{1}{128.962 \pm 0.016} \text {. }
$$

The difference with the result in ref. 20 is minute. (4.2) may be compared with a recent calculation of Hagiwara et al., ${ }^{[21]}$ who, using only experimental $e^{+} e^{-}$annihilation data at low energy find

$$
\bar{\alpha}_{\text {Q.E.D. }}\left(M_{Z}^{2}\right)=\frac{1}{128.954 \pm 0.031} .
$$

While the central value is compatible with ours, the error in (4.3) is twice as large. This shows clearly the advantage of a thorough use of analyticity as well as of combining several sets of data (we will discuss this further in Sect. 6, in connection with the muon anomaly).

4.2. The masses and widths of the rho, the quadratic radius and second coefficient of the pion, and the scattering length and effective range parameter for the $\mathrm{P}$ wave in $\pi \pi$ scattering

As other byproducts of our analysis we can give very precise numbers for the masses and widths of the neutral and charged rho resonances, for the quadratic radius and second coefficient of the pion form factor, and for the the scattering length, $a_{1}^{1}$, and effective range parameter, $b_{1}^{1}$ (defined as in ref. 9), for the $\mathrm{P}$ wave in $\pi \pi$ scattering. In what regards the first, we have the following results: for the charged rho, and in $\mathrm{MeV}$,

$$
\begin{array}{ccc} 
& \text { TY, } \tau, e \pi, e^{+} e^{-} & \text {GJ }(\tau \text { decay only }) \\
m_{\rho^{ \pm}} & 774.0 \pm 0.4 & 775.4 \pm 0.4 \\
\Gamma_{\rho^{ \pm}} & 147.7 \pm 0.7 & 149.3 \pm 0.4 \\
\chi^{2} \text { d.o.f. } & 1.06 & \sim 1.8 .
\end{array}
$$

For the neutral rho,

$$
\begin{array}{cccc} 
& \mathrm{TY}, e^{+} e^{-}, e \pi & \mathrm{TY}, \tau, e \pi, e^{+} e^{-} & \mathrm{GJ}\left(e^{+} e^{-} \text {only }\right) \\
m_{\rho^{0}} & 773.1 \pm 0.6 & 773.2 \pm 0.4 & 772.95 \pm 0.7 \\
\Gamma_{\rho^{0}} & 141.7 \pm 1.2 & 146.0 \pm 0.8 & 147.9 \pm 0.7 \\
\chi^{2} \text { d.o.f. } & 0.9 & 1.06 & \sim 1.7
\end{array}
$$

Here TY are our results here, and GJ refers to the results by Ghozzi and Jegerlehner. ${ }^{[22]}$ Clearly, the masses are stable and well determined, the widths less so.

The quadratic radius and second coefficient of the pion are defined by

$$
F_{\pi}^{2}(t) \underset{t \rightarrow 0}{\simeq} 1+\frac{1}{6}\left\langle r_{\pi}^{2}\right\rangle t+c_{\pi} t^{2}
$$

and our results allow a precise determination of both quantities. The same is true for $a_{1}^{1}$, 
$b_{1}^{1}$. We have, for $\pi^{+} \pi^{-}$,

$$
\begin{array}{cccc} 
& \text { TY }\left[\text { Only } e^{+} e^{-}, e \pi\right] & \text { TY [Including } \tau \text { decay] } & \text { Colangelo } \\
\left\langle r_{\pi}^{2}\right\rangle\left(\mathrm{fm}^{2}\right) & 0.423 \pm 0.003 & 0.432 \pm 0.001 & 0.435 \pm 0.005 \\
c_{\pi}\left(\mathrm{GeV}^{-4}\right) & 3.78 \pm 0.05 & 3.84 \pm 0.02 & - \\
a_{1}^{1}\left(m_{\pi} \equiv 1\right) & (38.9 \pm 2.3) \times 10^{-3} & (37.8 \pm 0.8) \times 10^{-3} & (37.9 \pm 0.5) \times 10^{-3} \\
b_{1}^{1}\left(m_{\pi} \equiv 1\right) & (4.1 \pm 0.7) \times 10^{-3} & (4.74 \pm 0.09) \times 10^{-3} & (5.67 \pm 0.13) \times 10^{-3}
\end{array}
$$

TY is from our results here and "Colangelo" from refs. 8, 9. The discrepancy between the result for $b_{1}^{1}$ from the pion form factor and that in ref. 9 had already been noted in ref. 10 . The numbers coming from our calculations for $a_{1}^{1}, b_{1}^{1}$, including tau decay data, agree much better than those not including it with results from pion-pion scattering, either from phase shifts analyses or using the Froissart-Gribov representation, ${ }^{[10]}$ which is another reason for preferring the results including tau decays.

The values of the parameters of our fits are

$$
\begin{aligned}
& e^{+} e^{-}, e \pi: \\
& c_{1}=0.26 \pm 0.04, \quad c_{2}=0.19 \pm 0.13, \\
& b_{0}=1.106 \pm 0.009, \quad b_{1}=0.23 \pm 0.10
\end{aligned}
$$

and

$$
\begin{aligned}
& e^{+} e^{-}, e \pi, \tau: \\
& c_{1}=0.24 \pm 0.01, \quad c_{2}=-0.18 \pm 0.03, \\
& b_{0}=1.074 \pm 0.006, \quad b_{1}=0.13 \pm 0.04 .
\end{aligned}
$$

The numbers $b_{i}$ in (4.8) correspond to $\pi^{+} \pi^{-}$; they are the ones we have used to calculate $a_{\mu}\left(s \leq 0.8 \mathrm{GeV}^{2}\right)$. For $\pi^{0} \pi^{+}$we would have,

$$
b_{0}=1.064 \pm 0.006, \quad b_{1}=0.13 \pm 0.03 \quad\left[\pi^{0} \pi^{+}\right],
$$

and the $c_{i}$ are as in (4.8). The corresponding scattering length and effective range parameter are, in units of $m_{\pi^{+}}$,

$$
a_{1}^{1}=(37.8 \pm 0.8) \times 10^{-3}, \quad b_{1}^{1}=(4.78 \pm 0.09) \times 10^{-3} \quad\left[\pi^{0} \pi^{+}\right] .
$$

\section{Comparison of our theoretical calculations with experiment for $a_{\mu}$}

We return to the magnetic moment of the muon, and present, in Table I, a summary of our results for $a$ (Hadr.). In this Table we have added our old result from 2002, ref. 6, and the result of a recent evaluation by Jegerlehner, ${ }^{[21]}$ in which theory is kept to a minimum; for example, only data are used for $s<0.8 \mathrm{GeV}^{2}$, and QCD is taken valid only for energies above $13 \mathrm{GeV}$. The main interest of this type of calculation lies in its role as control of the calculations where a more comprehensive use of theory (as well as extra experimental information) is made. In the Table we also include the recent experimental value of ref. 2. 
For our evaluations here, we have separated explicitly the estimated errors due to radiative corrections, and the light-by-light scattering diagram.

\begin{tabular}{|c|c|c|}
\hline & $10^{11} \times a_{\mu}\left(s \leq 0.8 \mathrm{GeV}^{2}\right)$ & $10^{11} \times a_{\mu}(\mathrm{Hadr})$. \\
\hline Jegerlehner & - & $6840 \pm 94$ \\
\hline $\mathrm{TY}(2002), e^{+} e^{-}, e \pi, \tau$ & $4774 \pm 51$ & $6993 \pm 69$ \\
\hline $\mathrm{TY}, e^{+} e^{-}, e \pi$ & $4715 \pm 32 \pm 10(\mathrm{rad})$. & $6935 \pm 50 \pm 10(\mathrm{rad}.) \pm 30(\ell \times \ell)$ \\
\hline $\mathrm{TY}, e^{+} e^{-}, e \pi, \tau$ & $4798 \pm 31 \pm 10(\mathrm{rad})$. & $7018 \pm 49 \pm 10(\mathrm{rad}.) \pm 30(\ell \times \ell)$ \\
\hline Experiment & & $7209 \pm 60$ \\
\hline
\end{tabular}

\section{TABLE I}

Contributions to the rho region, and to the hadronic part of the muon anomaly. Jegerlehner: ref. 22. TY (2002): ref. 6. TY: this article.

From Table I it is clear that there is reasonable agreement among the various theoretical determinations, but there is a definite distance between the central values from theory and experiment, at a level between $2.3 \sigma$ and $3.3 \sigma$, if we add quadratically "rad" and " $\ell \times \ell$ " errors to the other ones. In the remaining of this section we will discuss possible reasons for this discrepancy.

An obvious reason would be new physics; we will not discuss this here, since it lies outside the scope of the present paper, and send the interested reader to the hundreds of papers that have been written discussing this possibility.

A second reason is, of course, a displacement of the experimental result. Since the experimental number in Eq. (1.1) comes basically from only one experiment, it could happen that an independent determination would move it to better agreement with the results of the theoretical evaluations.

And a third possibility is that the central values of some of the theoretical evaluations presented here are displaced with respect to the true values. The more obvious place where such a displacement may occur is the evaluation of $a(\ell \times \ell)$. The two approximations used to evaluate this contribution do not have overlapping ranges of validity; there is a wide region, when the virtualities of some (one) of the intermediate photons are small, and at the same time other (others) are large, where neither the one-pion or the constituent quark approximations need to be valid. In fact, the only certain result we have on this piece is the coefficient of the leading chiral $\operatorname{logarithm}\left(\log ^{2} m_{\pi}\right)$ given in ref. 23, which is of little practical use. However, it is not easy to see how one could get the large values necessary for theory and experiment to overlap. In a recent calculation, using methods somewhat different to previous ones, Melnikov and Vainshtein ${ }^{24]}$ find $10^{11} \times a(\ell \times \ell)=136 \pm 25$. Although this is larger by a bit more than one sigma $\left(44 \times 10^{-11}\right)$ than the result quoted in (3.3), it is not sufficient to remove the discrepancy. 
Finally, we may have a coincidence of several of the effects mentioned here, with the bad luck that they add.

\section{Comparison with other recent calculations and concluding remarks}

Our analysis shows that, to get a precise value of $a_{\mu}$, it is certainly necessary to profit from the existence of methods that allow us to make full use of theory, in particular for fitting the pion form factor: the use of robust theory in the fits produces robust results. It is not very consistent to use analyticity and unitarity to write the representation (2.1), and refuse to use exactly the same ingredients to improve the knowledge of $F_{\pi}$. In this sense, it is also important to take into account the data on $F_{\pi}(s)$ for spacelike $s$, i.e., from $e \pi$ scattering. This has been deemed inappropriate by some authors because they (may) contain systematic errors. However, if one refrained from taking into account data afflicted by systematic errors, there would be no data one could use. Systematic errors can and should be taken into account as shown, in this particular instance, in ref. 6 and in the present article.

In the same vein, we believe that tau decay data can and should be used, in spite of the fact that the data of the various tau decay experiments differ in some energy regions by more than one standard deviation -doubtlessly because of systematic errors, as is obvious from (2.11c). This is particularly important because the errors given in Eq. (2.8a) for $a_{\mu}^{(2)}\left(s \leq 0.8 \mathrm{GeV}^{2}\right)$ are deceptively small. As we already commented, the error per experimental point for $e^{+} e^{-} \rightarrow \pi \pi$, fitting only $e^{+} e^{-}, e \pi$ data, is $89 / 113 \simeq 0.79$, clearly smaller than unity; while, even imposing tau decay information, the error per experimental point is (as reported in Eq. (2.11c)) only of $108 / 113 \simeq 0.97$, perfectly acceptable. This means that acceptable fits -like, indeed, the one obtained by us using also tau decay datacan be found outside the nominal error bars in Eq. (2.8a). ${ }^{6}$

Likewise, when data on $F_{\pi}$ from the processes $e^{+} e^{-} \rightarrow \pi^{+} \pi^{-}+\gamma$ are forthcoming, they should be incorporated into the analysis: the safest way to get rid of systematic errors is to combine data of various, independent experiments, so that the various independent systematic errors average out. The gain, both in accuracy and robustness that follows from our methods can perhaps be seen more clearly if we compare them with other recent evaluations, ${ }^{[21,25,26]}$ something that we do in Table II (where we do not include the results of ref. 22, already discussed before). Here the stability of our 2002 results against including new, more precise $e^{+} e^{-}$data, contrasts with the variations in the other determinations, is spite of the fact that our error is substantially smaller.

In short: fitting to the theoretical expressions instead of integrating directly the data, allows us to compare the different data samples among them in a fully quantitative

\footnotetext{
${ }^{6}$ One should, however, not forget that the value we needed for the tau decay normalization error, $(-1.4 \pm 0.5) \%$ (cf. Eq. (2.11b)), or $(-1.2 \pm 0.5) \%$ if including the estimate $(2.16)$ for $\delta_{\gamma}$, is slightly larger than the expected normalization error, as given by the Particle Data Tables, $0.7 \%$.
} 
THE HADRONIC CONTRIBUTIONS TO THE ANOMALOUS MAGNETIC MOMENT OF THE MUON

\begin{tabular}{|c|c|c|}
\hline & $10^{11} \times a_{\mu}^{\mathrm{had}, \mathrm{LO}+\gamma}$ & $10^{11} \times a_{\mu}$ \\
\hline Ezhela $\left[e^{+} e^{-}\right]$ & $6996 \pm 89$ & $116591835 \pm 96$ \\
\hline Hagiwara $\left[e^{+} e^{-}\right]$ & $6924 \pm 64$ & $116591763 \pm 74$ \\
\hline Davier (a) $\left[e^{+} e^{-}\right]$ & $6847 \pm 70$ & $116591693 \pm 78$ \\
\hline Davier (b) $\left[e^{+} e^{-}\right]$ & $6963 \pm 72$ & $116591809 \pm 80$ \\
\hline Davier (b) $[\tau]$ & $7110 \pm 58$ & $116591956 \pm 68$ \\
\hline TY (2002), $e^{+} e^{-}, e \pi, \tau$ & $7002 \pm 66$ & $116591849 \pm 69$ \\
\hline TY, $e^{+} e^{-}, e \pi, \tau$ & $7027 \pm 49$ & $116591889 \pm 58$ \\
\hline Experiment & & $116592080 \pm 60$ \\
\hline
\end{tabular}

TABle II

The lowest order hadronic part of the muon anomaly, including photon corrections [Eq. (3.9)], and the full $a_{\mu}$. Hagiwara: ref. 21. Ezhela: ref 25. Davier, (a) and (b): ref. 26, (a) and (b). TY (2002): ref. 6. TY: this article.

manner. We believe that only using this kind of quantitative comparisons one can decide if the suggested discrepancies are meaningful or only apparent, and, if meaningful, if they are due to systematic errors or to physics. The fact is that one can fit all the $e \pi, e^{+} e^{-} \rightarrow \pi \pi$ and tau data with a $\chi^{2} /$ d.o.f. essentially 1 . This result is a non-trivial improvement on previous work.

It is, however, not clear to us that one can improve the results using $\pi \pi$ scattering data. If we include in the fit the experimental numbers for $\delta_{1}^{1}$, the value of $a_{\mu}$ increases by $8 \times 10^{-11}$; but this is not necessarily more precise than the result without including this information. The systematic errors of $\delta_{1}^{1}$, due to the fact that one does not scatter real pions (and thus one has to rely on models), are larger than the errors in our calculation. And if, like Colangelo and collaborators, ${ }^{[8,9]}$ we input $\delta_{1}^{1}$ from theoretical analyses (Roy equations and chiral perturbation theory), one is depending on determinations whose accuracy has been challenged ${ }^{[10]}$ and is, very likely, too optimistically estimated.

Apart from this, to get real improvement in the theoretical predictions for the quantity $a_{\mu}$ (Hadr.) it would be, first of all, necessary to remove the sources of uncertainty mentioned at the end of Sect. 5. Of these, the one stemming from $\ell \times \ell$ is unlikely to be removed in a satisfactory manner; and we have also a problem (although less important numerically) with electroweak radiative corrections to tau decay. If we treat them by considering the pions as elementary, and factoring out $F_{\pi}$, then Sirlin's theorem ${ }^{[12]}$ implies that, for $\tau \rightarrow \nu \pi \pi$, the logarithmic piece, $\log M_{Z} / m_{\tau}$ cancels out. It, however, does not cancel if we consider that, at short distances, the decay is really $\tau^{-} \rightarrow \nu \bar{u} d$. This means that the model with elementary pions fails, for this case of tau decay, and thus that the 


\section{J. F. DE TROCÓNIZ AND F. J. YNDURÁIN}

chances of removing the uncertainties due to lack of accurate knowledge of the radiative corrections here are remote.

As a final comment, we would like again to bring attention to the mismatch between the experimental and theoretical values for $a_{\mu}$; although not yet definite proof of failure of the standard model, it cannot be ignored. 
THE HADRONIC CONTRIBUTIONS TO THE ANOMALOUS MAGNETIC MOMENT OF THE MUON

\section{Acknowledgements}

We are grateful to Fred Jegerlehner for discussions - oral and e-mail- that have helped a lot to clarify important issues.

\section{References}

1 The QED calculations of $a_{e}$, and (some of) those of $a_{\mu}$, with references, may be found in the review of V. W. Hughes and T. Kinoshita, Rev. Mod. Phys. 71, S133 (1999).

2 H.N. Brown et al., Phys. Rev. Letters 86, 2227 (2001); G. W. Bennett et al., Phys. Rev. Letters 89, 101804 and (E) 129903 (2002); G. W. Bennett et al., hep-ex/0401008 v3.

3 For the electromagnetic corrections, see the text by T. Kinoshita et al., Quantum Electrodynamics, World Scientific, 1990 and T. Kinoshita and M. Nio, hep-ph/0402206, whose numbers we take. Weak corrections were first calculated by R. Jackiw and S. Weinberg, Phys. Rev. D5, 2396 (1972); I. Bars and M. Yoshimura, ibid. D6, 374 (1972); K. Fujikawa, B. W. Lee and A. I. Sanda, ibid 2932 (1972). The hadronic weak corrections have been recently reconsidered M. Knecht et al., JHEP 0211, 003 (2002), and a detailed reassessment of all electroweak contributions is that by A. Czarnecki, W. J. Marciano and A. Vainshtein, Phys. Rev. D67, 073006 (2003).

4 Novosibirsk, $\rho$ region: L. M. Barkov et al., Nucl. Phys. B256, 365 (1985); R. R. Akhmetshin et al., Budker INP 99-10 (1999) hep-ex/9904027, superseded by R. R. Akhmetshin et al., Phys. Letters B527, 161 (2002). The details of the calculations of the radiative corrections to $e^{+} e^{-} \rightarrow \pi \pi$ scattering, necessary to extract the pion form factor from data, are given in P. Singer, Phys. Rev. 130, 2441 (1963) and Erratum, 161, 1694 (1967) and A. B. Arbuzov et al., J. High Energy Phys. 10, 1 and 6 (1997).

5 Novosibirsk, $\omega$ and $\phi$ region, $K K$ and $3 \pi$ : R. R. Akhmetshin et al., Phys. Letters B466, 385 and 392 (1999); ibid., B434, 426 (1998) and ibid., B476, 33 (2000); hep-ex/0308008 (2003). M. N. Achasov et al., Phys. Rev. D63, 072002 (2001); M. N. Achasov et al., Phys. Letters 365 B462 (1999) and Preprint Budker INP 98-65 (1998) hep-ex/9809013]. $\phi \rightarrow 2 \pi$ : M. N. Achasov et al., Phys. Letters B474, 188 (2000).

6 J. F. de Trocóniz and F. J. Ynduráin, Phys. Rev. D65, 093001 (2002).

7 S. R. Amendolia et al., Nucl. Phys. B277, 168 (1986).

8 H. Leutwyler, hep-ph/0212324; G. Colangelo, hep-ph/0312017.

9 G. Colangelo, J. Gasser, and H. Leutwyler Nucl. Phys. B603, 125, (2001).

10 J. R. Peláez and F. J. Ynduráin, Phys. Rev. D68, 074005 (2003) and FTUAM 03-19 (2003) hep-ph/0312187.

11 ALEPH: R. Barate et al., Z. Phys. C76, 15 (1997); OPAL: K. Ackerstaff et al., Eur. Phys. J. C7, 571 (1999); CLEO: S. Anderson et al., Phys. Rev., D61, 112002 ((2000).

12 A. Sirlin, Nucl. Phys. B196, 83 (1982); W. J. Marciano and A. Sirlin, Phys. Rev. Lett. 61, 1815 (1988). 


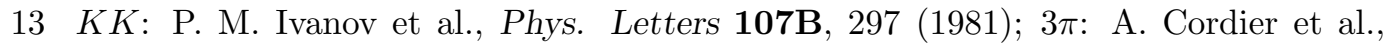
Nucl. Phys. B172, 13 (1980); $4 \pi$ and more: G. Cosme et al., Nucl. Phys. B152, 215 (1979). [For a review, see S. Dolinsky et al., Phys. Reports C202, 99 (1991), and other work quoted there]. All these references give results for energies below $s=2 \mathrm{GeV}^{2}$. Between 2 and $9 \mathrm{GeV}^{2}$, see C. Bacci et al., Phys. Letters 86B, 234 (1979). At the $\bar{c} c$ threshold region: J. Z. Bai et al., Phys. Rev. Lett., 88, 1010802 (2002).

14 For the QCD expressions: K. G. Chetyrkin, A. L. Kataev and F. V. Tkachov, Phys. Letters B85, 277 (1979); M. Dine and J. Sapiristein, Phys. Rev. Lett. 43, 668 (1979); W. Celmaster and R. Gonsalves, Phys. Rev. Lett. 44, 560 (1980); S. G. Gorishny, A. L. Kataev and S. A. Larin, Phys. Letters B259, 144 (1991); L. R. Sugurladze, and M. A. Samuel, Phys. Rev. Lett. 66, 560 (1991). s quark mass: S. Chen et al., Eur. J. Phys. C22, 31 (2001); b, $c$ quark masses: A. Pineda and F. J. Ynduráin, Phys. Rev. D58, 094022 (1998) and Phys. Rev. D61, 077505 (2000). $\alpha_{s}$ : F. Le Diberder and A. Pich, Phys. letters B289, 165 (1992); J. Santiago and F. J. Ynduráin, Nucl. Phys. B563, 45 (1999) and Nucl. Phys. B611, 447 (2001). For a review, see S. Bethke, J. Phys. G26, R27 (2000) and, for the direct measurement of $\alpha_{s}$ on the Z, D. Strom, "Electroweak measurements on the $Z$ resonance", Talk presented at the 5th Int. Symposium on Radiative Corrections, RadCor2000, Carmel, Ca., September 2000.

15 S. Narison, Phys. Letters B513, 53 (2001) and (E) B526, 414 (2002).

16 J. Bijnens, E. Pallante and J. Prades, Nucl. Phys. B474, 379 (1996); M. Hayakawa, T. Kinoshita and A. I. Sanda, Phys. Rev. D54, 3137 (1996); M. Hayakawa and T. Kinoshita, Phys. Rev. D57, 465 (1998).

17 M. Knecht and A. Nyffeler, Phys. Rev. D65, 073034 (2002).

18 B. Krause, Phys. Letters B390, 392 (1997)

19 P. Singer, Phys. Rev.130, 2441 (1963) and (E), 161, 1694 (1967).

20 J. F. de Trocóniz and F. J. Ynduráin, Phys. Rev. D65, 093002 (2002).

21 K. Hagiwara et al., Phys. Rev. D69, 093004 (2004).

22 F. Jegerlehner, Proc. Int. Frascati Conf., 2003 hep-ph/0310234; S. Ghozzi and F. Jegerlehner, Phys. Letters B583, 222 (2004).

23 Knecht et al., Phys. Rev. Lett. 88, 071802 (2002).

24 K. Melnikov and A. Vainshtein, FTPI-MINN-03-36 (hep-ph/0312226).

25 V. V. Ezhela, S. B. Lugovsky and O. V. Zenin, IHEP-2003-35 (hep-ph/0312114).

26 (a) M. Davier et al., Eur. Phys. J. C27, 497 (2003); (b) M. Davier et al., Eur. Phys. J. C31, 503 (2003). 